\title{
Femtosecond nonadiabatic dynamics in photosynthetic light harvesting
}

\author{
Peter W. Foster ${ }^{1}$, Vivek Tiwari ${ }^{1}$, William K. Peters ${ }^{1}$, and David M. Jonas ${ }^{1, *}$ \\ ${ }^{1}$ Department of Chemistry, University of Colorado, Boulder, CO 80309-0215 USA
}

\begin{abstract}
Fast and efficient energy transfer in photosynthetic antennas supports all life on earth. Nonadiabatic energy transfer drives unusual vibrations through tight coupling with electronic motion. Polarization dependent vibrational motion drives polarization independent femtosecond energy transfer.
\end{abstract}

\section{Introduction}

Photosynthesis supports essentially all life on earth. In photosynthesis, light is harvested by antennas containing thousands of light absorbing pigment molecules and transferred to reaction centers, which initiate a series of reactions that store energy by synthesizing high energy molecules. Energy transfer[1] and the primary charge separation[2, 3] are ultrafast and extraordinarily efficient, inspiring many investigations of their mechanism. Two-dimensional spectroscopy has revealed unusual quantum beat signatures for both processes,[4] which were initially hypothesized to arise from protein protection of electronic coherence. Recent experiments[5] support an alternative mechanism[1] in which vibrations that are delocalized over more than one pigment are amplified by coupling to electronic motion. We report unusual aspects of such electronically amplified vibrations.

\section{Model and calculation}

In the model we have used, intramolecular vibrations of the pigments become delocalized by the energy transfer coupling between excited states. Eq. (1) shows the vibrationalelectronic interactions for the very simplest Hamiltonian of this type, which describes a dimer in which two coupled pigments have unequal electronic excitation energies separated by a gap $\Delta$.

$$
\hat{H}_{\mathrm{int}}=(1 / 2) \omega\left(\hat{q}_{-}^{2}+\hat{p}_{-}^{2}\right) \hat{I}_{1}+\left[\begin{array}{cc}
\left(-\Delta / 2-\omega d \hat{q}_{-} / \sqrt{2}\right)|A\rangle\langle A| & J|A\rangle\langle B| \\
J|B\rangle\langle A| & \left(+\Delta / 2+\omega d \hat{q}_{-} / \sqrt{2}\right)|B\rangle\langle B|
\end{array}\right]
$$

The two electronic states with one pigment excited $(A$ and $B$ ) have a coordinate independent coupling $J$ that causes partial electronic delocalization. These delocalized

\footnotetext{
* Corresponding author: david.jonas@colorado.edu
} 
electronic states are known as Frenkel excitons. The pigment vibrations are also delocalized. The delocalized, anti-correlated vibration of the pigment pair, $q_{-}=\left(q_{A}-\right.$ $\left.q_{B}\right) / 2^{1 / 2}$, is coupled into the electronic dynamics because it tunes the pigment energy gap. The strongest coupling occurs when the vibrational frequency is resonant with the energy gap between delocalized electronic states; this causes a breakdown of the BornOppenheimer approximation that assumes vibrations and electronic motion are separable.

\section{Results}

Figure 1 shows the coupled vibrational and electronic dynamics for electronic excitation of the donor.

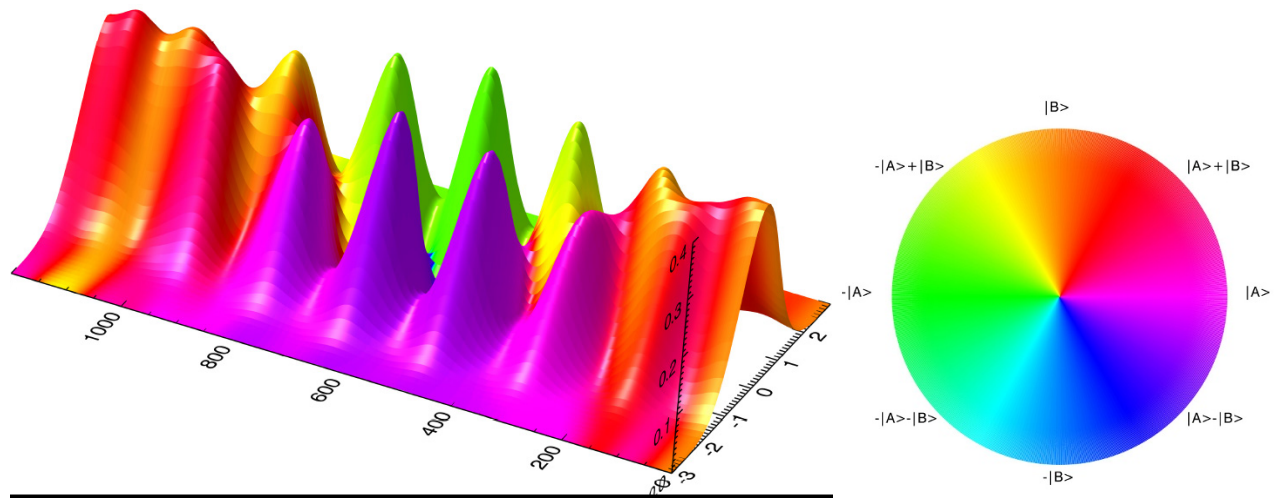

Fig. 1. Left) Vibrational probability density as a function of the anticorrelated coordinate and time over 1200 fs. Color indicates the electronic character through the color wheel (right). Here, the donor pigment $B$ is excited at $t=0$.

The parameters for Fig. 1 were chosen to mimic one pair of excitons and their resonant vibration in the highly efficient FMO antenna protein.[1] Figure 1 shows that progressive changes in electronic character are accompanied by a vibration of progressively larger amplitude. The energy transfer reaches a maximum at about $600 \mathrm{fs}$, when the electronic character is that of the acceptor A (bright green or purple) and switches sign from $|\mathrm{A}\rangle$ to $\mid A>$ (purple to green) as the vibrational wavepacket oscillates from side to side with each vibrational half period. Because this model does not include additional vibrations, the energy then goes back to the donor and the vibration dies away. This indicates the ideal damping through vibrational relaxation or vibronic decoherence should occur on a $600 \mathrm{fs}$ timescale. Interestingly, this matches the timescale for vibronic dephasing reported[5] in a recent $2 \mathrm{D}$ experiment on $\mathrm{FMO}$ at a temperature of $77 \mathrm{~K}$. Investigations of this damping process are under way.

The vibrational dynamics are polarization dependent. Figure 2 shows that the direction of vibration depends on the polarization of the exciting light in the molecular frame of the dimer. Typically, vibrations are excited through a change in equilibrium bond length or angle upon electronic excitation; this determines the direction and amplitude of vibrational motion (Franck-Condon principle). Figure 2 shows not only that the vibrational amplitude grows in time (as seen in Figure 1), but also that the initial direction of the vibrational motion is polarization dependent. This type of vibrational motion underlies the appearance of vibrational quantum beats in $2 \mathrm{D}$ spectra with the double-crossed polarization configuration $(-\pi / 4, \pi / 4, \pi / 2,0) .[1,5]$ Interestingly, although the vibrational dynamics vary with excitation polarization, the electronic dynamics always steadily maximize population on the lowest adiabatic 
electronic states of the acceptor at $600 \mathrm{fs}$. At present, this amazing feature of the coupled vibrational and electronic dynamics is only partially understood, but it could help optimize the energy transfer yield.

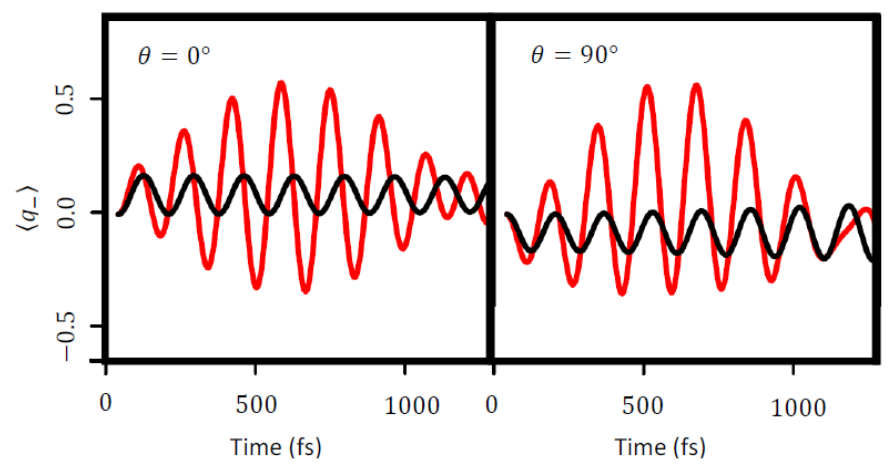

Fig. 2. Average vibrational wavepacket coordinates for two different excitation polarizations in the molecular frame. Left) Polarization at 0 drives initial vibrational motion to positive coordinates. Right) Polarization at 90 drives initial vibrational motion to negative coordinates. For both panels, the motion that results from the adiabatic approximation is shown in black and nonadiabatic wavepacket motion is shown in red. The larger amplitude vibration in red illustrates the electronic amplification of coupled vibrational motion during energy transfer.

\section{Conclusions}

Signatures of vibrational-electronic resonance have been found in femtosecond 2D spectra for many light harvesting proteins, and also in the reaction center of higher plants. This study has revealed tightly coupled vibrational and electronic motions that generate these signatures at liquid nitrogen temperatures. Fluctuations and damping at physiological temperature[6] are under investigation.

This material is based upon work supported by the National Science Foundation under Grant No. CHE-1405050. Any opinions, findings, and conclusions or recommendations expressed in this material are those of the authors and do not necessarily reflect the views of the National Science Foundation.

\section{References}

1. V. Tiwari, W. K. Peters, and D. M. Jonas, Proc. Natl. Acad. Sci. USA 110, 1203 (2013).

2. F. D. Fuller, J. Pan, A. Gelzinis, V. Butkus, S. S. Senlik, D. E. Wilcox, C. F. Yocum, L. Valkunas, D. Abramavicius, and J. P. Ogilvie, Nat. Chem. 6, 706 (2014).

3. E. Romero, R. Augulis, V. I. Novoderezhkin, M. Ferretti, J. Thieme, D. Zigmantas, and R. van Grondelle, Nat. Phys. 10, 677 (2014).

4. D. M. Jonas, Annu. Rev. Phys. Chem. 69, 327 (2018).

5. E. Thyrhaug, R. Tempelaar, M. A. Alcocer, K. Zídek, D. Bína, J. Knoester, T. L.C. Jansen, and D. Zigmantas, Nat. Chem. 10, 780 (2018).

6. H-G. Duan, V. I. Prokhorenko, R. J. Cogdell, K. Ashraf, A. L. Stevens, M. Thorwart, R. J. D. Miller, Proc. Natl. Acad. Sci. USA 114, 8493 (2017). 